\title{
Espécies cecidógenas (Diptera, Cecidomyiidae) e parasitóides (Hymenoptera) associadas a Guapira opposita (Vell.) Reitz. (Nyctaginaceae) na Restinga da Barra de Maricá, Rio de Janeiro
}

\author{
Valéria Cid Maia ${ }^{1}$ \\ Ricardo F. Monteiro ${ }^{2}$
}

\begin{abstract}
Gall-forming species (Diptera, Cecidomyiidae) and parasitoids (Hymenoptera) associated with Guapira opposita (Vell.) Reitz. (Nyctaginaceae) at Restinga of Barra de Maricá, Rio de Janeiro. Three different galls of Bruggmannia spp. (Diptera, Cecidomyiidae) on Guapira opposita (Vell.) Reitz. (Nyctaginaceae) were studied at Restinga of Barra de Maricá, State of Rio de Janeiro. Thirty parasitoid species are associated to these galls. Most of them is generalist and solitary. Hyperparasitoidism is common. The trophic relations among gall makers and parasitoids are presented.

KEY WORDS. Cecidomyiidae, Bruggmannia, galls, Hymenoptera, Guapira, parasitoid
\end{abstract}

A Restinga da Barra de Maricá, Estado do Rio de Janeiro, é um ambiente bastante rico em galhas, conforme observado por MONTEIRO et al. (1994). Estes autores registraram para a região 60 tipos de galhas de Cecidomyiidae, em 25 famílias de plantas. Dentre estas, destacaram-se as Myrtaceae e Nyctaginaceae pela diversidade de galhas.

Recentemente, MAIA \& COURI (1996) estudaram as galhas nas Myrtaceae da Restinga da Barra de Maricá, caracterizando-as morfologicamente e identificando as espécies cecidógenas.

As galhas de Nyctaginaceae carecem de mais estudo. MONTEIRO et al. (1994) registraram oito tipos de galhas de Cecidomyiidae nesta família de planta, todos em Guapira opposita (Vell.) Reitz, na Restinga da Barra de Maricá e de Arraial do Cabo, Rio de Janeiro. Deste montante, seis tipos já haviam sido previamente estudados por COURI \& MAIA (1992), MAIA (1993) e MAIA \& COURI (1993). Tais autoras identificaram e descreveram as espécies indutoras e caracterizaram as galhas. Dos dois tipos restantes, o galhador de um deles é aqui registrado pela primeira vez - Bruggmannia sp. (provavelmente trata-se de uma nova espécie, porém devido à dificuldade de obtenção dos adultos, a espécie ainda não foi descrita) e o outro permanece desconhecido.

1) Museu Nacional. Quinta da Boa Vista, São Cristóvão, 20940-040 Rio de Janeiro, Rio de Janeiro. Bolsista da CAPES.

2) Departamento de Ecologia, Centro de Ciências da Saúde, Instituto de Biologia, Universidade Federal do Rio de Janeiro. 21944-970 Rio de Janeiro, Rio de Janeiro, Brasil. 
MONTEIRO et al. (1994) registraram ainda a alta freqüência de galhas com parasitóides (Hymenoptera) na Restinga de Barra de Maricá.

Este trabalho restringue-se às galhas induzidas por Bruggmannia elongata Maia \& Couri, 1993; B. robusta Maia \& Couri, 1993 e Bruggmannia sp., e tem como objetivo fornecer dados sobre a abundância dos galhadores, registrar os parasitóides mais freqüentes e estabelecer as relações tróficas entre os mesmos.

\section{MATERIAL E MÉTODOS}

No período de fevereiro/1988 a janeiro/1989, foram realizadas excursões quinzenais à Restinga da Barra de Maricá, para estudar no campo a abundância das espécies galhadoras e para coletar galhas, visando a criação em laboratório dos insetos a elas associados.

Dados sobre a abundância dos indutores foram obtidos a partir da contagem das galhas presentes em 10 espécimens da planta hospedeira. Para cada espécimen, foi contado o número dos diferentes tipos de galhas estudados em 100 folhas, totalizando portanto 1000 folhas em cada excursão.

Foi calculado o número médio de galhas, somando os valores encontrados em cada mês e dividindo o total pelo número de meses.

Para a criação dos insetos galhadores e seus parasitóides: quinzenalmente, foram vistoriados dez espécimens da planta hospedeira, retirando 45 galhas (15 de cada tipo) por espécimen, sempre que a abundância das galhas o permitiu.

O material coletado foi transportado em sacos plásticos fechados para o laboratório de Ecologia de Insetos (Instituto de Biologia, Universidade Federal do Rio de Janeiro), onde, com auxílio de um esteremicroscópio, realizou-se a dissecção das galhas, observando desta forma os imaturos que se desenvolviam no interior das mesmas e seus hábitos. Após a dissecção, os diferentes tipos de galhas foram acondicionados individualmente em recipientes de vidro fechados, forrados por papel umedecido e etiquetados.

Os Hymenoptera obtidos foram conservados em álcool a 70\% e depositados no laboratório de Ecologia de Insetos da Universidade Federal do Rio de Janeiro. Parte dos Cecidomyiidae foram montados em lâminas, seguindo a metodologia de GAGNÉ (1994) e o excedente foi preservado em álcool 70\%. Os Cecidomyiidae foram incluídos na coleção de Diptera do Museu Nacional, Rio de Janeiro.

\section{RESULTADOS E DISCUSSÃO}

Sobre as galhas. Bruggmannia elongata induz a formação de galhas parenquimáticas, uniloculares e verdes. Bruggmannia robusta induz galhas globosas, vermelhas ou amarelas, uniloculares e preferencialmente na superfície inferior das folhas. Estas galhas estão descritas e ilustradas em MAIA \& COURI (1993). Bruggmannia sp. induz galhas cônicas, verdes e uniloculares. Estas galhas sobressaem em ambas as faces da folha: na face superior na forma de uma saliência arredondada e na inferior como uma projeção triangular. A galha madura apresenta opérculo na face superior, através do qual se dá a emergência do cecidógeno. 
Sobre a abundância das galhas e galhadores: os três tipos de galhas estudados ocorreram em todos os meses do ano. Para as galhas de $B$. elongata encontrou-se o número máximo de 2300 galhas mensais registrado em maio; o menor valor obtido foi de 750 galhas (em janeiro), e o valor médio mensal foi de 1323 galhas. Para $B$. robusta, os valores máximo, mínino e médio registrados foram os seguintes: 1320 (em agosto), 120 (em maio) e 387; para Brugggmannia sp.: 110 (em agosto), 8 (em junho) e 35,6. Em todos os meses do ano, os valores encontrados para $B$. elongata foram superiores aos de B. robusta e estes por sua vez superiores aos de Bruggmannia sp.

Sobre a freqüência e riqueza dos parasitóides: no total, foram encontradas 30 espécies diferentes de Hymenoptera associados às galhas estudadas. Vinte e quatro foram consideradas ocasionais (número de indivíduos menor que dez e ocorrência limitada a 1,2 ou 3 meses do ano) e seis espécies foram consideradas freqüentes (número de indivíduos maior ou igual a dez e ocorrência em pelo menos quatro meses do ano).

Das 30 espécies, 26 estão associados às galhas de $B$. elongata (seis freqüentes e 20 ocasionais); 11 às de $B$. robusta (cinco freqüentes e seis ocasionais) e três às de Bruggmannia sp. (ocasionais). Encontrou-se entre os parasitóides representantes de oito famílias diferentes: Eulophidae, Eurytomidae, Platygasteridae, Eupelmidae, Elasmidae, Signophoridae, Braconidae e Mymaridae, destacando-se as duas primeiras pela riqueza de espécies. A intensidade de ataque foi maior em imaturos de Bruggmannia elongata, espécie galhadora mais abundante e com maior riqueza de parasitóides associados (Tab. I).

Tabela I. Riqueza de espécies das familias dos parasitóides em galhas de Bruggmannia elongata, B. robusta e Bruggmannia sp. (Diptera, Cecidomyiidae) e taxa de parasitismo das espécies galhadoras, na Restinga da Barra de Maricá, Rio de Janeiro.

\begin{tabular}{lccc}
\hline \multicolumn{1}{c}{ Parasitóides } & B. elongata & B. robusta & Bruggmannia sp. \\
\hline Eulophidae & 14 & 5 & 1 \\
Eurytomidae & 7 & 2 & 2 \\
Platygasteridae & 2 & 2 & 0 \\
Eupelmidae & 1 & 0 & 0 \\
Elasmidae & 1 & 0 & 0 \\
Signophoridae & 1 & 0 & 0 \\
Braconidae & 0 & 1 & 0 \\
Mymaridae & 0 & 1 & 0 \\
Total & 26 & 11 & 3 \\
Taxa de parasitismo $(\%)$ & 43,5 & 27,0 & 1,0 \\
\hline
\end{tabular}

Sobre os hábitos dos himenópteros: neste estudo, foram encontradas seis espécies de parasitóides freqüentes: Chrysonotomyia sp., Platygaster sp., Rileya sp., Entedoninae sp., Eurytoma sp. e Galeopsomopsis sp. (Tab. II). Deste total, dois são ectoparasitas: Platygaster sp. e Galeopsomopsis sp. e quatro são endoparasitas: Chrysonotomyia sp., Rileya sp., Entedoninae sp. e Eurytoma sp.. A grande maioria mostrou-se solitária, exceção apenas para Platygaster sp., cujo gregarismo parece estar relacionado à poliembrionia. Ainda sobre Platygaster sp., deve-se ressaltar o 
seu comportamento como ectoparasitóide - fato pouco comum em espécies do mesmo gênero, onde predomina o endoparasitismo.

Tabela II. Hábitos das espécies dos parasitóides mais freqüentes de Bruggmannia elongata, B. robusta e Bruggmannia sp. (Diptera, Cecidomyiidae), na Restinga da Barra de Maricá, Rio de Janeiro.

\begin{tabular}{lcl}
\hline \multicolumn{1}{c}{ Parasitóides } & Hábito & \multicolumn{1}{c}{ Hospedeiro } \\
\hline Rileya sp. & $1 / 2$ & Bruggmannia elongata \\
& $1 / 2$ & Bruggmannia robusta \\
Eurytoma sp. & 1 & Bruggmannia sp. \\
& 1 & Bruggmannia elongata \\
Chrysonotomyia sp. & 1 & Bruggmannia sp. \\
& $1 / 2$ & Bruggmannia elongata \\
Platygaster sp. & $1 / 2$ & Bruggmannia robusta \\
& 3 & Bruggmannia robusta \\
Galeopsomopsis sp. & 3 & Bruggmannia elongata \\
& 4 & Bruggmannia robusta \\
Entedoninae sp. & 4 & Bruggmannia sp. \\
. & 4 &
\end{tabular}

(1) endoparasita isolado da larva galhadora; (2) hiperparasita isolado; (3) ectoparasita isolado ou gregário da larva galhadora; (4) ectoparasita isolado da pupa galhadora.

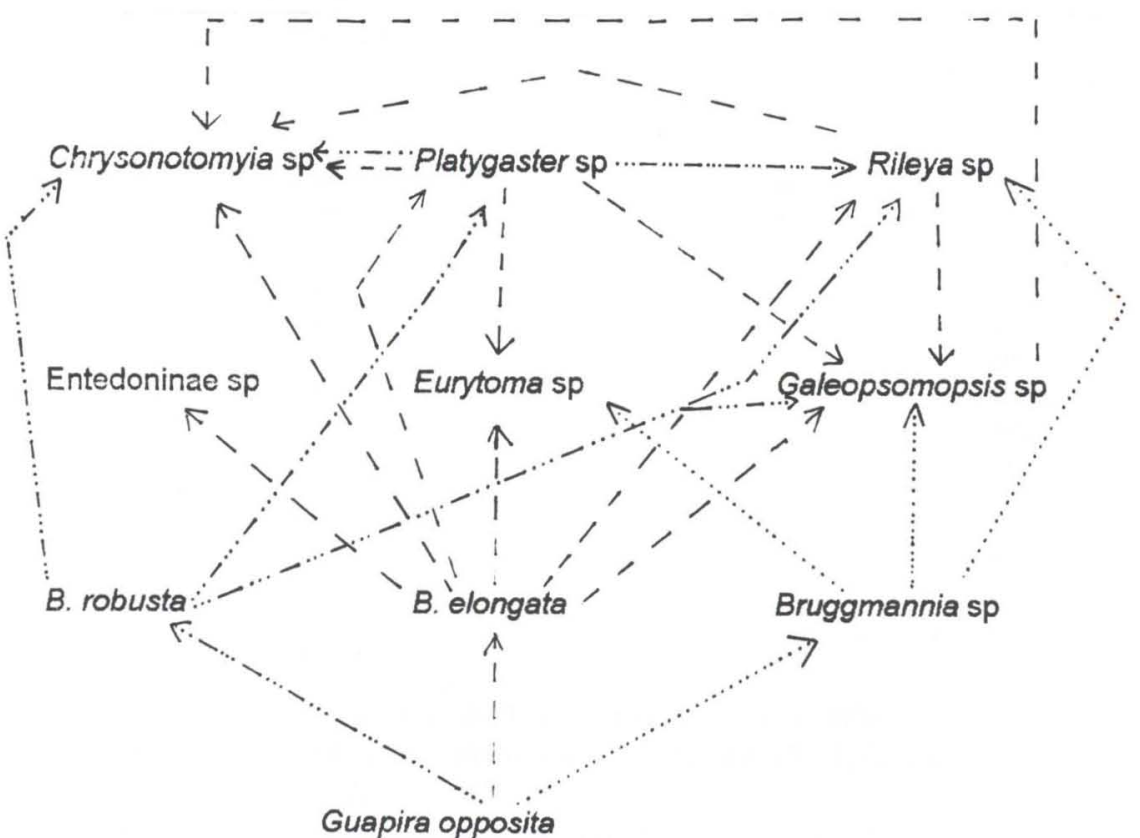

Fig. 1. Teia alimentar obtida entre Bruggmannia elongata, B. robusta e Bruggmannia sp. (Diptera, Cecidomyiidae) e seus respectivos parasitóides (Hymenoptera), em Guapira opposita (Nyctaginaceae), na Restinga da Barra de Maricá, Rio de Janeiro. Relaçöes observadas em galhas: $(--)$ parenquimáticas, $(\cdots \cdots \cdots-)$ globosas, $(\cdots \cdots)$ cônicas. 
Quase todos os parasitóides estudados atacaram a fase larval do hospedeiro, exceto Galeopsomopsis sp. (ectoparasita pupal). Chrysonotomyia sp., Rileya sp., Eurytoma sp. e Galeopsomopsis sp. atuaram também como hiperparasitóides.

As relações tróficas entre os insetos galhadores e os parasitóides mais freqüentes associados a Guapira opposita resultaram em uma teia alimentar complexa (Fig. 1). A partir desta teia, pode-se observar que cinco espécies de Hymenoptera atacaram mais de uma espécie galhadora; apenas Entedoninae sp. limitou-se a uma única espécie galhadora. Das seis espécies estudadas, quatro atuaram também como hiperparasitóide, enquanto que Entedoninae sp. e Platygaster sp. apareceram associados exclusivamente a galhadores. Tendo em vista que a maioria dos parasitóides atacaram diferentes espécies hospedeiras (tanto cecidomiídeos, como outros himenópteros), podemos considerá-los como generalistas, exceto Platygaster sp. (especialista para Bruggmannia spp.) e Entedoninae sp. (especialista para B. elongata).

AGRADECIMENTOS. À Maria Antonieta Pereira de Azevedo (Departamento de Ecologia, Universidade Federal do Rio de Janeiro, Rio de Janeiro) pela identificação das famílias de Hymenoptera; e ao Biosystematics and Beneficial Insects Institute (Maryland, E.U.A.) pela identificação dos gêneros dos parasitóides.

\section{REFERÊNCIAS BIBLIOGRÁFICAS}

Couri, M.S. \& V.C. MAIA. 1992. Considerações sobre Pisphondylia Möhn, 1960

(Diptera, Cecidomyiidae, Asphondyliidi), com descrição de uma espécie nova do Brasil. Revta bras. Ent. 36 (4): 729-730.

GAGNÉ, R.J. 1994. The Gall Midges of the Neotropical Region. Ithaca, Comstock Cornell University Press, 352p.

MAIA, V.C. 1993. Considerações sobre Proasphondylia Felt (Diptera, Cecidomyiidae), com descrições de duas espécies novas associadas com Guapira opposita (Velloso) Reitz. (Nyctaginaceae). Revta bras. Zool. 10 (2): 215-218

MAIA, V.C. \& M.S. CouRI. 1993. Descrição de três espécies novas de Bruggmannia Tavares, 1906 (Diptera, Cecidomyiidae, Asphondyliidi) do Brasil associadas com Guapira opposita (Nyctaginaceae). Rev. Brasil. Biol. 53 (2): 209-215.

- 1996. Cecidomyiidae (Diptera) associated with Myrtaceae at Restinga of Barra de Maricá (RJ). Rev. Brasil. Biol. 57 (2): 227-230.

Monteiro, R.F.; F. Ferraz; V.C. MAiA \& M.A.P. AZEVEdo. 1994. Galhas entomógenas em restingas: uma abordagem preliminar. In: Atas do III Simpósio de Ecossistemas da Costa Brasileira, São Paulo, ACIESP 87, Vol. 3, p.: 210-220. 